\title{
Prenatal Sonographic Features and Postnatal Outcome of Congenital Posteromedial Bowing of Tibia: An Experience from a Tertiary Fetal Medicine Center
}

\author{
Indrani Suresh • Vijayalakshmi Raja • Vijay Sriram • \\ Surekha Ravulapalli • Sathya Lakshmi Balusamy • \\ Shanthi Sairam · Sujatha Jagadeesh · Latha Murlidhar
}

Received: 30 April 2014/Accepted: 21 May 2014/Published online: 11 July 2014

(C) Society of Fetal Medicine 2014

\begin{abstract}
A series of 18 fetuses were identified from the archives, between 2004 and 2013 with findings of unilateral shortening of the tibia and fibula, an acutely dorsiflexed foot and posteromedial bowing of the tibia and fibula. The median gestational age at diagnosis was 23 weeks (range 20-29 weeks). The discrepancy in length between the affected and contralateral tibia varied from 9 to $33 \%$. Data on pregnancy outcome were available for 14 (78\%) of 18 fetuses. Four patients opted for termination of pregnancy. All the live born babies (10/14) were followedup postnatally and were well at a median age of 28 months (6-63 months) with milestones appropriate for age at the time of writing. Spontaneous resolution was noted in all the cases and none of them required surgical correction for limb discrepancy so far. Congenital posteromedial bowing of the leg is a benign entity whose outcome includes spontaneous resolution of the angular deformity, albeit with some residual limb length discrepancy and ankle valgus which may need orthopedic intervention around
\end{abstract}

I. Suresh · V. Raja · S. Ravulapalli .

S. L. Balusamy $(\bowtie) \cdot$ S. Sairam

Department of Fetal Medicine, Mediscan Systems,

Chennai 600004, India

e-mail: sbsatthya@yahoo.com

\section{Sriram}

Department of Orthopaedics, Kanchi Kamakoti Childs Trust

Hospital, Chennai, India

S. Jagadeesh

Department of Genetics, Mediscan Systems, Chennai, India

L. Murlidhar

Department of Perinatal Pathology, Mediscan Systems, Chennai, India
6-7 years of age. Awareness of this condition helps in making the diagnosis prenatally and in counseling the couples about the prognosis and management options available.

Keywords Posteromedial bowing - Angulation tibia · Shortening of leg $\cdot$ Prenatal ultrasonography $\cdot$ Dorsiflexed foot

\section{Introduction}

Congenital posteromedial bowing of the tibia (CPMBT) and fibula is an unusual congenital anomaly of the lower limb. The exact etiology is not known. The diagnosis is usually made after birth when the newborn presents with an acutely dorsiflexed foot apposed to the shin. The posterior and medial bowing is confirmed on a radiograph (anteroposterior and lateral views). This deformity is known to resolve spontaneously. In some cases, residual limb length discrepancy and ankle valgus may be present, which will require surgical correction. The prenatal diagnosis of this condition has been first described by Zollinger et al. in 2002 [1]. The authors describe here, the prenatal ultrasound findings and outcome of this rare condition seen in a series of 18 patients.

\section{Report of Cases}

Cases with findings of unilateral shortening of tibia and fibula with dorsiflexed foot and bowing of the leg were included for the study. The authors identified 18 cases in 9 years (2004-2013) from the database. 

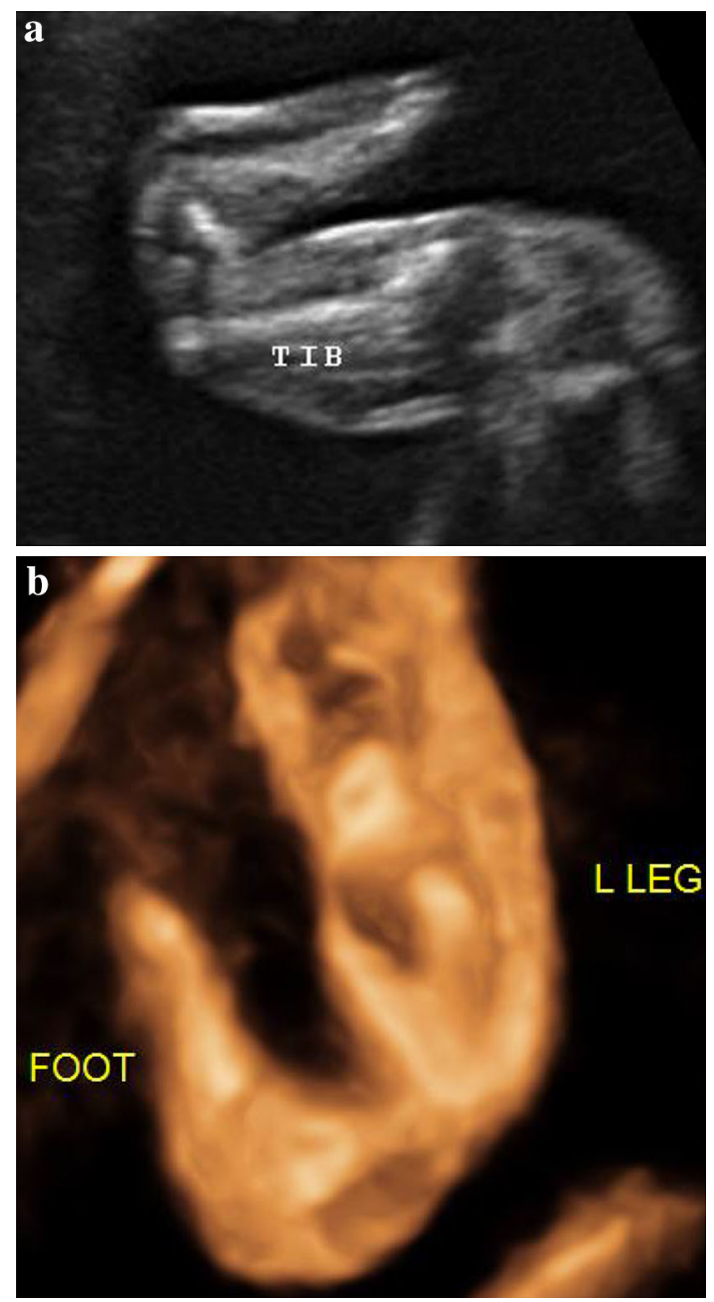

Fig. 1 a 2D image b 3D image showing bowing of tibia and a dorsiflexed foot

Being a tertiary fetal medicine centre, it caters to a mixed population comprising both screening and referral groups. Amongst the 18 cases, 6 (33\%) cases were referred for a mid-trimester target scan and the rest $12(67 \%)$ were specifically referred for a suspected limb anomaly which included talipes (1), rocker bottom feet (1), angulated foot (1) and unilateral shortening of limb (9) prenatal findings and postnatal outcome of cases with CPMBT are set in Table 1.

The median maternal age was 26 years (20-32 years). None of them had a consanguineous marriage, except for one with fourth degree consanguinity. Ninety-four percent (17/ 18) were primigravidae. No significant medical illness was present, except in two patients (one was on antiepileptic drugs and the other was a gestational diabetic with a dichorionic twin pregnancy). The husband of one of the patients had bilateral talipes deformity operated in childhood.

The median gestational age at diagnosis was 23 weeks (20-29 weeks). Prenatal consultation with a pediatric orthopedic surgeon led to the sonographic diagnosis of
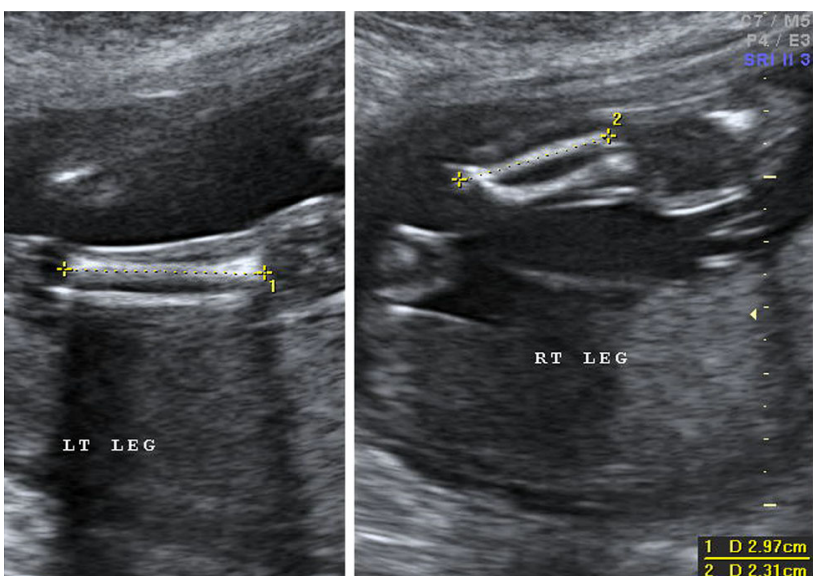

Fig. 2 Mesomelic shortening of right leg

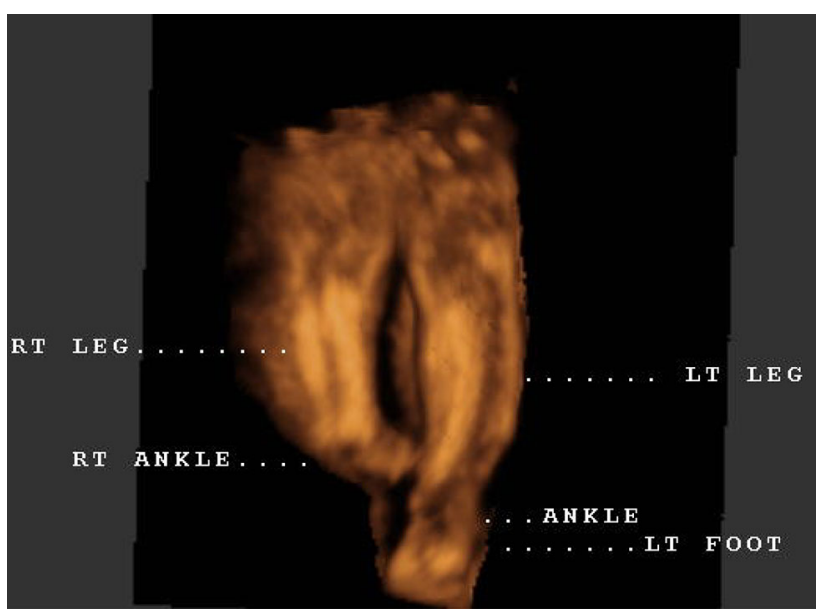

Fig. 3 3D image showing lower limb discrepancy

posteromedial bowing of tibia in the first case. Dorsiflexed foot nestled against the tibia (calcaneovalgus deformity) was the characteristic ultrasound finding in all the patients (Fig. 1). Apart from the posture, the foot was structurally normal. Shortening of the tibia (Figs. 2, 3) was identified in all, but the degree of shortening in comparison with the contralateral tibia varied, ranging from 0.3 to $1.5 \mathrm{~cm}$ $(9.3-33 \%)$. The fibula was invariably involved in all the cases.

The findings were unilateral and isolated. A detailed examination of the skeletal system included measurement of all the long bones, their contour and echogenicity. A detailed systematic examination of the rest of the skeletal system and other organ systems revealed no other identifiable anomalies except one case of unilateral renal agenesis. The families were counseled in detail with involvement of the pediatric orthopedic surgeon regarding prognosis and postnatal management. The recorded clippings of the children who 


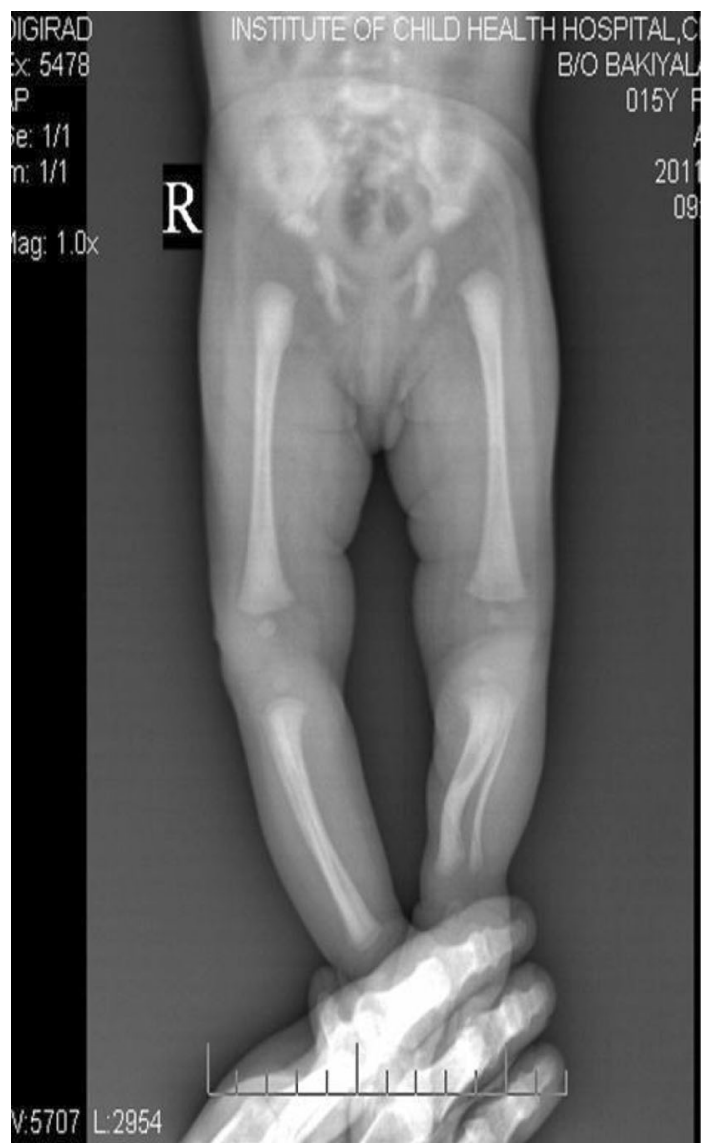

Fig. 4 Fetogram of a case with congenital posteromedial bowing of tibia

survived this condition were shown while counseling these families after obtaining consent from the concerned couples. Consent for publishing the photos had also been obtained.

Among the five patients who were serially followed-up at the centre, the tibial length discrepancy appeared to be maintained with advancing gestation in 4 patients compared to one where the discrepancy increased from 16 to $27 \%$. Data on pregnancy outcome were available for 14 (14/18) patients. Four patients opted for termination of pregnancy while autopsy was done for three cases. Findings were proven at autopsy and fetogram revealed the characteristic posteromedial angulation (Fig. 4). No other additional abnormalities were identified on autopsy. All live born babies (10/14) were followed-up postnatally. The diagnosis was confirmed and findings corroborated postnatally by clinical (Figs. 5,6 ) and radiological examination. The milestones of these children were appropriate for the given age. Median age of these children at the time of writing was 28 months (6-63 months). They were variously treated with splints, shoe and passive flexion to correct the calcaneovalgus. Surgical correction was not required for any of these babies till date.

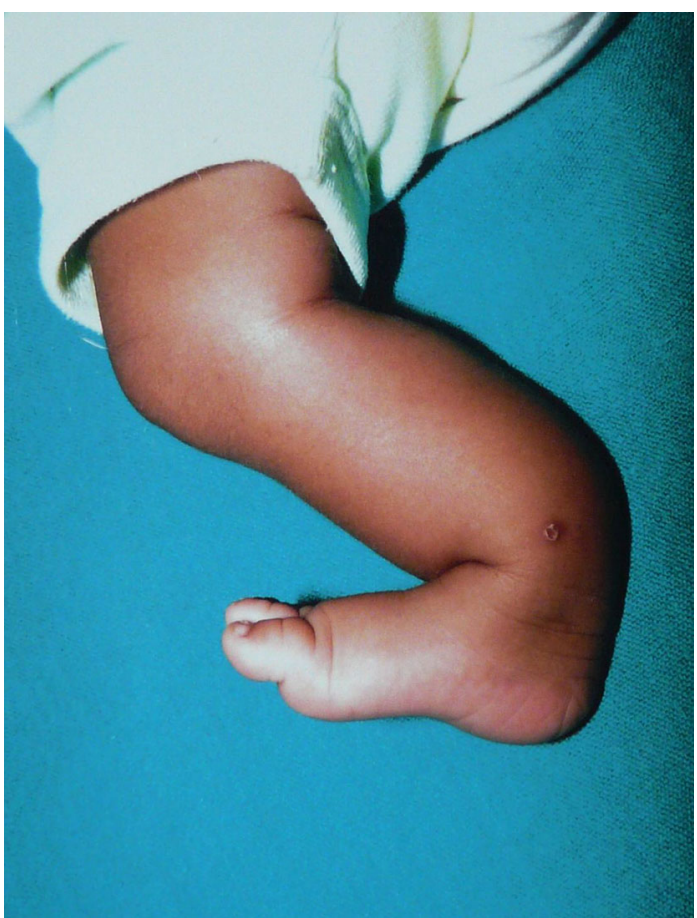

Fig. 5 Postnatal photo of the baby diagnosed with CPMBT antenatally

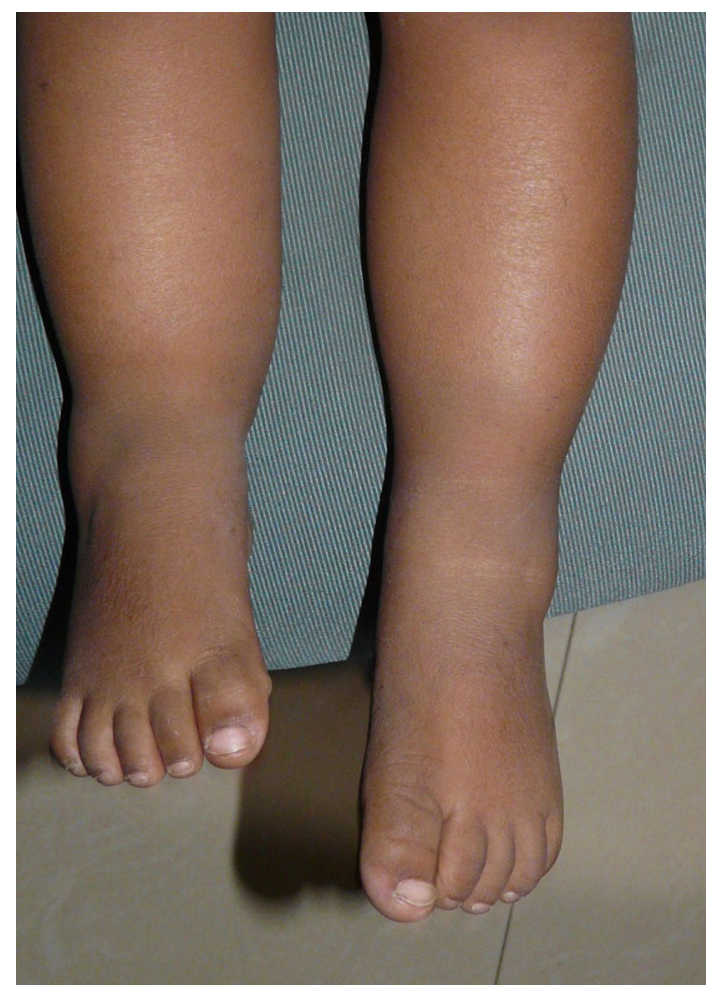

Fig. 6 Picture of the child at 1 year of age, diagnosed prenatally with CPMBT 
Table 1 Prenatal findings and postnatal outcome of cases with CPMBT

\begin{tabular}{|c|c|c|c|c|c|c|}
\hline Case & $\begin{array}{l}\text { Gestational age } \\
\text { at diagnosis (weeks) }\end{array}$ & Side & $\begin{array}{l}\text { Tibial } \\
\text { shortening }(\mathrm{cm})\end{array}$ & $\begin{array}{l}\text { Dorsiflexed } \\
\text { foot }\end{array}$ & Posterior bowing & Outcome \\
\hline 1. & 23 & $\mathrm{Rt}$ & $0.4(11.8 \%)$ & + & + & Male-63 months \\
\hline 2. & 21 & $\mathrm{Rt}$ & $0.4(14 \%)$ & + & + & Male-30 months \\
\hline 3. & 23 & $\mathrm{Rt}$ & $0.6(16.2 \%)$ & + & + & Female-44 months \\
\hline 4. & 20 & $\mathrm{Rt}$ & $0.4(13.7 \%)$ & + & + & Male-41 months \\
\hline 5. & 21 & $\mathrm{Lt}$ & $0.3(9.3 \%)$ & + & + & Female-29 months \\
\hline 6 & 20 & $\mathrm{Rt}$ & $0.6(20.7 \%)$ & + & + & Female-30 months \\
\hline 7. & 26 & $\mathrm{Lt}$ & $1.1(27 \%)$ & + & + & Female-13 months \\
\hline 8. & 28 & $\mathrm{Lt}$ & $0.9(20 \%)$ & + & + & Male-12 months \\
\hline 9. & 22 & $\mathrm{Rt}$ & $0.9(20 \%)$ & + & + & Female-6 months \\
\hline 10. & 24 & $\mathrm{Lt}$ & $1(30 \%)$ & + & + & Male-10 months \\
\hline 11. & 24 & $\mathrm{Lt}$ & $0.8(23 \%)$ & + & + & \multirow{4}{*}{$\begin{array}{l}\text { Termination of pregnancy } \\
\text { Autopsy done for three. }\end{array}$} \\
\hline 12. & 25 & $\mathrm{Lt}$ & $0.4(11.8 \%)$ & + & + & \\
\hline 13. & 21 & $\mathrm{Rt}$ & $0.9(28 \%)$ & + & + & \\
\hline 14. & 25 & $\mathrm{Lt}$ & $1.5(33 \%)$ & + & + & \\
\hline 15. & 24 & $\mathrm{Rt}$ & $0.9(23.6 \%)$ & + & + & Lost to follow-up \\
\hline 16. & 29 & $\mathrm{Rt}$ & $1.3(31 \%)$ & + & + & \\
\hline 17. & 23 & $\mathrm{Rt}$ & $0.6(19.4 \%)$ & + & + & \\
\hline 18. & 22 & $\mathrm{Lt}$ & $1.0(27.7 \%)$ & + & + & \\
\hline
\end{tabular}

\section{Discussion}

Congenital posteromedial bowing of tibia is a rare anomaly characterized by bowing of tibia and fibula medially and posteriorly, usually in the middle or distal third of the shaft. This condition is almost always accompanied by an ipsilateral calcaneovalgus deformity. The exact etiology and the pathogenesis remains unknown. Majority of the cases are diagnosed postnatally. A prenatal series was reported by Zollinger et al. [1]. According to the authors, the present series appears to be the second and the largest reported ever.

The authors could not identify any predisposing factor for this condition in the present study. There is more predilection for the primigravidas. The median gestational age at diagnosis was 23 weeks (20-29 weeks) indicating that a substantial number of these cases can be identified in the routine mid-trimester target scan. This anomaly is usually unilateral as seen in the present series. The only case of bilateral CPMBT was reported by Shah et al. [2]. There was no sex predilection. In the present study, the right and left legs were almost equally involved in contrast to the more likely involvement of left side seen by Shah et al. [2].

Prenatally, measurement of the tibia and fibula is done with ease, compared to the assessment of degree of bowing. The initial appearance of the leg and foot in CPMBT in the newborn is often a cause for concern among parents and so is the appearance of the limb and foot on prenatal ultrasound to the unaware. The most striking feature on ultrasound which drew attention to the deformity was the characteristic posture of the foot which was acutely dorsiflexed and almost parallel to the tibia (calcaneovalgus deformity). The differential diagnosis included potentially all conditions which may present with short or bent bones [3].

The isolated nature of the abnormality, absence of involvement of the rest of the skeletal system, unilateral shortening of leg and the characteristic dorsiflexed foot on the affected side favored the diagnosis of CPMBT. Significant shortening and bowing was often self-evident but subtle degrees of shortening could be established only by measurement of the affected and contralateral tibia and fibula. The direct correlation between initial bowing and ultimate discrepancy in the limb length has been established in a number of studies $[2,4,5]$. However, in a recent review by Kaufman et al. [6], inhibition of growth at the distal tibial physis has been implicated as the primary factor in limb shortening. Two distinct mechanisms namely physeal realignment and diaphyseal remodeling has been attributed to the resolution of deformity [4].

Whether the degree of discrepancy seen prenatally can be correlated with the ultimate limb length discrepancy at skeletal maturity needs to be established by further follow up studies of these children. CPMBT is a condition which is known to resolve spontaneously, often to a large degree, within the first year of life $[4,5]$. 


\section{Conclusion}

Imaging and documentation of the extremities is a standard protocol in our institution during mid-trimester anomaly scan. The importance of examining the hands and feet cannot be over emphasized since they are often the clue to an underlying chromosomal abnormality or genetic condition. Nonetheless, these need to be distinguished from isolated limb abnormalities as the prognosis and hence counseling aspects can be very different. Awareness of this condition helps in counseling the couples when faced with these findings on the nature of the problem and the management options available.

\section{Conflict of interest None}

\section{References}

1. Zollinger PE, Wessels MW, Wladimiroff JW, Diepstraten AFM. Prenatal ultrasonographic diagnosis of posteromedial bowing of the leg: two case reports. Ultrasound Obstet Gynecol. 2000;15:150-3.

2. Shah HH, Doddabasappa SN, Joseph BJ. Congenital posteromedial bowing of the tibia: a retrospective analysis of growth abnormalities in the leg. J Pediatr Orthop B. 2009;18(3):120-8.

3. Cheema JI, Grissom LE, Harcke HT. Radiographic characteristics of lower-extremity bowing in children. Radiographics. 2003;23(4):871-80.

4. Pappas AM. Congenital posteromedial bowing of the tibia and fibula. J Pediatr Orthop. 1984;4(5):525-31.

5. Hofmann A, Wenger DR. Posteromedial bowing of the tibia Progression of discrepancy in leg lengths. J Bone Joint Surg Am. 1981;63(3):384-8.

6. Kaufman SD, Fagg JA, Jones S, Bell MJ, Saleh M, Fernandes JA. Limb lengthening in congenital posteromedial bow of the tibia. Strategies Trauma Limb Reconstr. 2012;7(3):147-53. 